\title{
Influence of Extreme Weather and Climate Change on the Resilience of Power Systems: Impact and Possible Mitigation Strategies
}

Mathaios Panteli, ${ }^{\mathrm{a}, *}$ and Pierluigi Mancarella ${ }^{\mathrm{b}}$

${ }^{\mathrm{a}, \mathrm{b}}$ School of Electrical and Electronic Engineering, The University of Manchester, Sackville Street, Manchester, M13 9PL, UK, emails: mathaios.panteli@manchester.ac.uk, p.mancarella@manchester.ac.uk Tel: +441613068721

\begin{abstract}
A key driver for developing more sustainable energy systems is to decrease the effects of climate change, which could include an increase in the frequency, intensity and duration of severe weather events. Amongst others, extreme weather has a significant impact on critical infrastructures, and is considered one of the main causes of wide-area electrical disturbances worldwide. In fact, weather-related power interruptions often tend to be of high impact and sustained duration, ranging from hours to days, because of the large damage on transmission and distribution facilities. Hence, enhancing the grid resilience to such events is becoming of increasing interest. In this outlook, this paper first discusses the influence of weather and climate change on the reliability and operation of power system components. Since modeling the impact of weather is a difficult task because of its stochastic and unpredicted nature, a review of existing methodologies is provided in order to get an understanding of the key modeling approaches, challenges and requirements for assessing the effect of extreme weather on the frequency and duration of power system blackouts. Then, the emerging concept of resilience is discussed in the context of power systems as critical infrastructure, including several defense plans for boosting the resilience of power systems to extreme weather events. A comprehensive research framework is finally outlined, which can help understand and model the impact of extreme weather on power systems and how this can be prevented or mitigated in the future.
\end{abstract}

Keywords: Climate change, Power Systems Blackouts, Reliability, Resilience, Resiliency, Weather 


\section{Introduction}

A key driver for developing more sustainable energy systems is to decrease the effects of climate change, which include occurrence of severe weather events. Amongst others, extreme weather such as hurricanes and storms is considered one of the main causes of wide-area electrical disturbances worldwide. In USA, for example, the annual impact of weather-related blackouts ranges from $\$ 20$ to $\$ 55$ billion [1] and the trend of such events shows that their frequency has increased over the last 30 years, with a dramatic increase in the 2000s. [2]. These disruptive events can be classified into five categories, namely, small impact, moderate, serious, major and extreme, based on the number of customers disconnected and the duration and frequency of the event [3]. In fact, severe weather caused approximately $80 \%$ of the largescale power outages from 2003-2012 [2]. Climate change is expected to further increase the frequency, intensity and duration of extreme weather events $[4,5]$, as a result mainly of the continuous rise in the global greenhouse gas (GHG) concentrations.

Electrical power systems are amongst the critical infrastructures of modern societies, so it is crucially important to boost their resilience to severe weather and to any future challenges that may arise due to the climate change. The future energy networks should go greener with reduced GHG emissions to mitigate the degree and impact of the climate change, but should also be resilient to unforeseeable external shocks, such as extreme weather. This leads to developing the so-called "low-carbon resilient" networks which combine both carbon reduction and resilience goals, as discussed in [6] and [7].

In this context, resilience is defined as the ability of a power system to withstand extraordinary and high impact-low probability events such as due to extreme weather, rapidly recover from such disruptive events and absorb lessons for adapting its operation and structure to prevent or mitigate the impact of similar events in the future [6,8-11]. Adaptation refers to the measures taken to reduce the vulnerability and build resilience [12] and it can be defined as "the process of adjustment to actual or expected climate and its effects, in order to moderate harm or exploit beneficial opportunities" [5].

However, building highly resilient networks to extreme weather events is a difficult task due to the high 
impact of such events to the transmission and distribution facilities. On the other hand, the so far low probability of occurrence of most such events makes it hard to develop a suitable cost benefit analysis. Also, uncertainty in the evolution of weather and potential change in the frequency or intensity of such extreme events makes the picture even more complex.

Modeling the influence of weather on the reliability of power systems has attracted the interest of several researchers and, as a result, numerous methodologies have been developed in the last decades. A review of these methodologies is presented in this paper, in order to understand the key modeling approaches, limitations and challenges in assessing the impact of weather on the frequency and severity of power system blackouts. In addition, a review of measures for improving the network resilience and the natural disaster emergency, preparedness and response is provided in this paper. These can be divided in short-term preventive and corrective measures, i.e., before (e.g. days or weeks), during and after a weather event; and long-term preventive measures which refer to planning and network reinforcement schemes for providing adaptability and robustness to future weather events and climate change.

Following this, a generic research framework is provided for enabling the development of weatherrelated resilience studies. In general, three models are required in such studies, namely, weather, component and system models. These models interact with each other to estimate the weather-affected system resilience indices.

This paper is organized as follows. Section 2 discusses the influence of severe weather and climate change on power system components. Section 3 provides the state-of-the-art techniques and challenges for assessing the effect of weather on power systems resilience. The defense plans to such events for boosting the network resilience are presented in Section 4. The proposed research framework is presented and discussed in Section 5. Section 6 summarizes and concludes the paper.

\section{Influence of Weather and Climate Change on Power System Components}

The extreme weather events have a significant influence on the reliability and operation of electrical components, and in turn on the resilience of the entire power infrastructure, the extent of which depends on 
the severity of the weather $[13,14]$ :

- High temperatures and heat waves limit the transfer capability of transmission lines, and increase the energy losses and the line sagging.

- High winds during storms and hurricanes can lead to faults and damage to overhead transmission and distribution lines, either by debris being blown against the lines or even a tower collapse in extremely high winds.

- Cold waves, heavy snow and the accumulation of ice can also cause failures of overhead lines and towers. Under freezing conditions, ice and snow may gather on insulators, which bridge the insulators and provide a conducting path, resulting in a flashover faults.

- Lightning strikes on or near overhead conductors can also cause short-circuit faults, which will trigger the electrical protection and the disconnection of the lines. Such faults are usually transient and are thus rapidly restored to service. However, the voltage surge caused by the strike may be transferred along the line and cause damage to equipment, such as transformer wings.

- Rain and floods do not pose a danger to overhead transmission lines, but to substation equipment, such as switchgear and control cubicles. The combination of rain with strong wings or lightning can however be significant threat to overhead lines.

It can be seen that the impact of severe weather can be direct destructive events, such as tower collapses due to high winds, or indirect events affecting the normal operation of electrical components, such as heat and cold waves.

Further, an important aspect that affects the degree of the influence of weather is the condition of the components. It is expected that aged components are more likely to be more vulnerable to extreme weather conditions than newer components. In fact, the exposure of power systems to severe weather conditions is often amplified by over-aged or degraded transmission and distribution equipment $[15,16]$.

The climate change is expected to have a great impact on the weather parameters discussed above and consequently on the operation and reliability of power systems [17-24]. The operation of several 
components, such as transformers and overhead lines, is governed by the maximum permissible operating temperature. An increase in the ambient temperature will thus affect their operating ratings and possibly limit their capabilities as the system elements would need to be derated to cope with the higher temperatures. Also, thermal power plants will be affected by the decreasing efficiency of the thermal conversion due to the rising ambient temperatures [23]. In addition, an increase in the temperature will affect the efficiency of the substation earthing, as the earthing impedance changes in response to the ground's moisture and temperature [18]. The rise in the sea level will threaten the coastal assets such as oil and gas pipelines, while the increased rainfall will increase the probability of floods. Changes in precipitation patterns as well as higher frequency and intensity of drought periods may adversely impact hydropower generation and affect water availability for cooling purposes in thermal and nuclear power plants. The efficiency of the other renewable energy resources will also be affected by the climate change [17]. For example, an anticipated increase in cloudiness will affect the efficiency of solar technologies [23] and wind generation may have to be shut down when extremely high winds are experienced. Severe weather conditions due to climate change can also result to fuel supply interruptions, which affect the availability of primary energy resources [12]. Furthermore, the climate change is also thought to contribute to catastrophic wildfires around the world, such as United States and Canada, as a result of longer and warmer seasons, which threaten mainly the transmission facilities. In addition, the growth in the demand and change in load patterns due to the weather and climate change [25-29], in combination with the population migration to areas most affected by the climate change or most prone to weather-related hazards, will create major bottlenecks in delivering electrical energy efficiently and reliably [21].

Based on these discussions, it is clear that extreme weather events can significantly impact the critical power infrastructure in several ways, but assessing this impact requires further studies to quantify possible changes in their frequency and intensity due to changes in the climate. If the rate of this change is lower than the rate of the development of power systems, then the power system should be able to adapt to the change in the weather pattern. However, if the rate of climate change is higher, then it may impose a threat 
to the resilience of power systems because they have been traditionally designed to be robust for relatively normal and stable weather and loading patterns [21]. Nevertheless, replacing, uprating or making all the power system components more robust to cope with the potentially increased impact of severe weather events due to the climate change is a very expensive and rather unrealistic solution. More efficient and cost-effective short-term and long-term planning would therefore be required to boost the resilience of power systems to such events.

\section{Assessing the Effect of Weather on Power System Operation and Resilience Implications: State- of-the-Art}

Modeling the influence of weather on the reliability and operation of power system components and in turn on the resilience of the entire power infrastructure is a difficult task due to the stochastic and unpredicted nature of weather. This section provides a review of the existing methodologies in this research area for identifying the key modeling approaches, challenges and limitations.

\subsection{Modelling techniques}

The techniques used in power systems reliability assessment can be generally divided into analytical and Monte Carlo simulations. Analytical techniques are preferred for small-scale system configurations because of their simplicity and low computational burden, but the simulation techniques are more suitable when complex system operational conditions are modelled.

The majority of the methods in the literature for evaluating the weather-associated impact on power systems reliability use analytical techniques, with Markov approach being the dominant analytical technique. The basic concepts of incorporating weather-related failures were first introduced in [30] and [31]. In [30], a two-state weather model is proposed for modeling the failure bunching phenomenon in parallel facilities (Figure 1.a). The "failure bunching due to adverse weather" refers to the sharp increase in the failure rate of the components and in the probability of overlapping component failures during highly stressed conditions, such as adverse weather. This must not be confused with the common mode or common cause failures [32]. Markov process is used by Billinton and Bollinger in [31] to evaluate the 
impact of weather on simple parallel configurations, where the two-state weather model is used to illustrate the bunching effect. The two-state weather model is the most widely used weather model [33-36], mainly because of its simplicity. Using this model, the weather conditions are divided in two states: normal and adverse. The failure and restoration rates are considered different but constant in each weather state. This results in two sets of reliability indices which can be weighted by the probability of transition between the two weather states. Liu and Singh [35] propose a DC Optimal Power Flow (OPF) Markov cut-set method (MCSM), which is based on the concept of applying the two-state weather model and the Markov process only to the minimal cut sets resulting in load shedding in order to estimate their reliability indices, and not to all system components. The proposed MCSM is compared with a next event sequential simulation (NESS), concluding that the analytical method is fast and of comparable accuracy with the NESS. Billinton and Wenyuan [37] use the two-state weather model and the Monte Carlo sampling technique to incorporate the variable weather conditions in composite analysis.

However, as argued in [38], the reliability indices obtained using the two-state weather model can be very optimistic. As a result, a third weather state, i.e. major adverse weather, has been added in [32] and [39] based on IEEE Standard 346 [40] to better capture the variations in weather severity (Figure 1.b). A generalized state space diagram for a Markov multi-state weather model is proposed in [41], where it is argued that even the three-state weather model may be inadequate to represent the wide and fluctuating range of weather conditions.

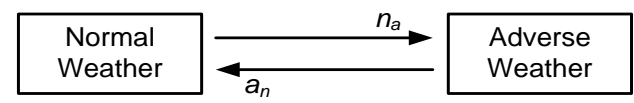

(a) Two-state weather model

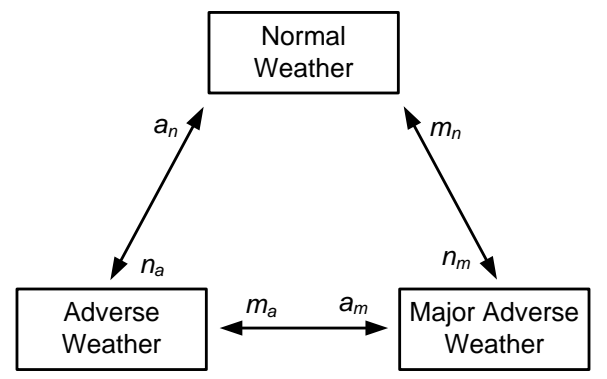

(b) Three-state weather model

Figure 1: Markov Weather Models 
Other analytical techniques used in the existing literature include the Bayesian networks [42, 43], and in particular the noisy OR-gate model [44], the fuzzy clustering techniques for mapping the relation between hurricane parameters and the increment multipliers of failure rates (IMFR) of transmission lines [45], and the Boolean Logic Driven Markov process for assessing the reliability of small-scale smart grids under variable weather conditions [46].

Nevertheless, because of the stochastic and space- and time-dependent nature of the weather events and the size and complexity of real power systems, the simulation techniques are considered more suitable than the analytical techniques for weather-related power system resilience studies. These simulation techniques must be capable to capture the traversing nature of the weather events, both space- and time-wise, and also model their stochastic impact on the operational and reliability parameters of power system components as they move across the network. The failure rates of the outdoor power system components depend on the weather conditions and might increase with the weather intensity level, which is highly stochastic. In addition, the restoration time of the damaged components, e.g. overhead lines and transmission towers, also depends on the weather conditions, which can be significantly longer than the restoration time during normal weather. Therefore, using multiple weather states with fixed failure and restoration times is quite inflexible and not practical for the resilience assessment of large power systems.

Monte Carlo simulation is applied by Rios et al. [47] to evaluate the impact of time-dependent phenomena and weather conditions on the computation of value of security (VaS) in power systems. Two main weather states are considered: normal and adverse. A weather-dependent failure rate is then determined based on the duration of each weather state and the proportion of failures for each component in each weather state. A sequential Monte Carlo approach is used in [33] for modeling the traversing weather events, taking into account their speed and direction. The Time To Fail (TTF) and Time To Repair (TTR) are resampled when the weather enters a new state using a failure rate multiplier and a repair rate divider for incorporating the weather impact. However, only one direction and fixed moving speed are considered, which does not represent the real nature of weather events. Time-varying failure rates are proposed by 
Wang and Billinton in [48] using time-varying weather weight factors. These are then inserted in a timesequential approach (TSDISREL99) for evaluating the system reliability indices, concluding that timevarying failure rates result in an increase in the unreliability cost indices for frequency sensitive load and a slight increase for the others. A non-homogenous Poisson process (NHPP) is used in [49] and [50] for generating the number and time of occurrence of high wind and lightning events, which are, however, considered standstill and not traversing. Then, similarly to [48], time-varying failure and restoration rates are developed and inserted in a sequential Monte Carlo approach to determine the influence of the weather stochasticity on the reliability indices. Li et al. [51] use historical weather data and outage records from Northeast USA utilities for developing a wind storm probability model and parameterizing the weatherdependent component models. Following this, sequential Monte Carlo is used for evaluating the performance of an actual distribution system in Northeast USA under six different wind storm intensity categories, ranging from normal to severe hurricanes.

\subsection{Consideration of weather regions}

It is usually assumed that the system is exposed to the same weather conditions at any given time by modeling weather as a standstill event. This reduces the complexity of the modeling procedure because no regional weather aspects are considered. This is a valid assumption for distribution systems which cover a small geographical area and the system is usually exposed to the same weather conditions at any given time. However, it is not applicable in transmission system resilience studies, which cover a large geographical area and the weather event moves across the transmission network sequentially with time. Billinton and $\mathrm{Li}$ [37] show that this assumption leads to the overestimation of the problem and to the estimation of pessimistic inadequacy indices.

As discussed in the existing literature, such as [37], [44] and [45], it is more accurate and practical to divide the network in weather regions. An arbitrary division of the IEEE 9-bus reliability test system into two weather regions is shown in Figure 2. The weather is assumed the same within each region, but it traverses and changes both time-wise and space-wise, i.e. region to region. Using weather regions helps 
model a weather event in a more realistic way and also estimate the regions' resilience to weather fluctuating events.

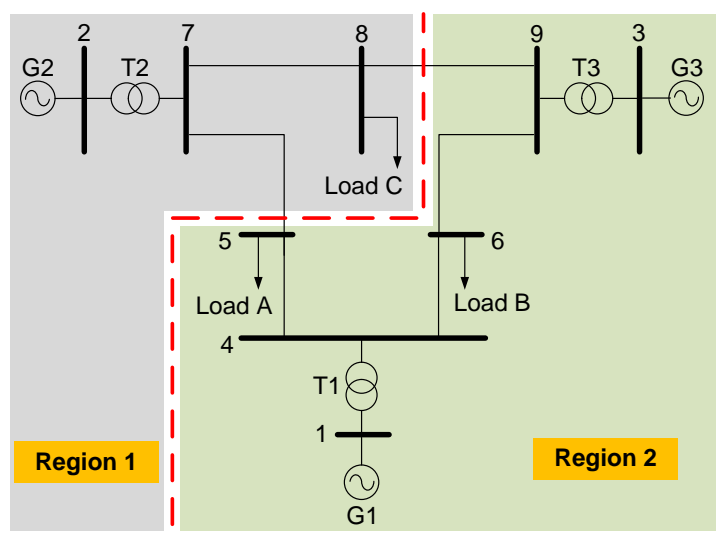

Figure 2: Dividing an electrical network in weather regions

Different criteria can be used for defining these weather regions, such as voltage levels [45] and meteorogical and geographical characteristics [37]. Splitting the system in weather regions based on the voltage levels is not realistic, but it simplifies the analysis. Ideally, the network will be divided based on real, recorded weather data, with the probability and duration of each weather state in each weather region given by meteorogical records. The number of weather regions may depend more on the availability of such data than on the size of the transmission network.

\subsection{Modeling the impact of weather on the failure rate of power system components}

The environment in which the transmission and distribution facilities reside has a significant impact on their reliability, and in particular on their failure rate. In addition, it is possible that a long transmission line crosses several weather regions, experiencing different weather conditions at each region. For example, lines 5-7 and 8-9 of Figure 2 cross the two weather regions. Therefore, the impact of weather across the transmission line will vary from region to region. Defining the weather-affected failure rate of the components with high accuracy is vitally important in modeling the impact of weather on the entire power infrastructure.

In [33], the line is divided into segments which are assumed to be connected in series. A failure rate multiplier is applied to each line segment, based on the fraction of the line in each weather region and on a 
failure stress factor for the segments in adverse weather. The line overall failure rate is then given by the summation of the failure rates of the line segments, since they are assumed to be in series. Liu and Singh [45] use the weighted average method to define an incremental multiplier of failure rate (IMFR) for each line segment, which depends on the portion of the line in each weather region. Barben [52] assumes that each line segment can fail independently and that they are designed to avoid the propagation of mechanical or electrical outages to the adjacent segments. Therefore, the line overall failure is simply given by the summation of the failure rates of the line segments.

An additional approach is to estimate the failure rate of each segment in each weather region and then choose the worst (highest) failure rate as a representative for the line. Also, the concept of fragility curves [53-56] can be used, which express the relation of the failure probability of any system component, such as transmission lines and towers or distribution poles, to any individual or combination of weather parameters, such as wind speed and lightning density. The shape of the curves depends on the impact of the weather parameter on the failure probability of the component. These fragility curves would be generic for each weather parameter and would correspond to all the weather regions. The regional weather profile would then be mapped to these curves for obtaining the regional weather-affected failure probabilities of the components (Figure 3). These curves can be developed either through modeling taking into account the weather aspects that affect the components' failure rate, such as wind speed, height and direction, or using real empirical data for determining the failure rate of the components under different weather conditions.

A strong assumption made in the methods discussed above is that a weather condition terminates at the boundary between two geographical weather regions. The division of the network in weather regions is based on the same assumption. However, in real systems, there are no clear frontiers between weather regions, but the weather event moves across the network with a fluctuating density, speed and duration, affecting the reliability of the electrical components in unpredictable ways. Nevertheless, dividing the network in weather regions and assuming same weather in each region provides a simple but effective way for assessing the impact of different weather conditions across the transmission lines. 


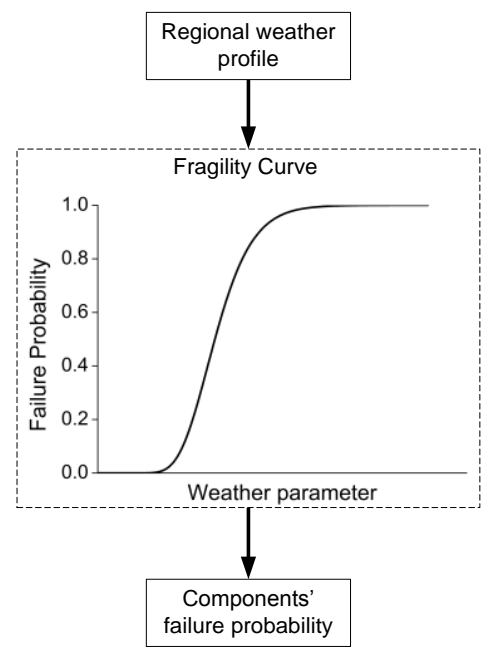

Figure 3: Determining components' failure probability using fragility curves

\subsection{Influence of weather on restoration time}

Figure 4 shows the restoration procedure of the weather-damaged components and the restoration of the power system to normal operation. Following the weather event, data need to be collected to assess the damage and set the priorities to initiate the system restoration. Then, the system operators coordinate with the repair crews for restoring the damaged components, followed by the corrective actions by the system operators for restoring the power system to a normal operation.

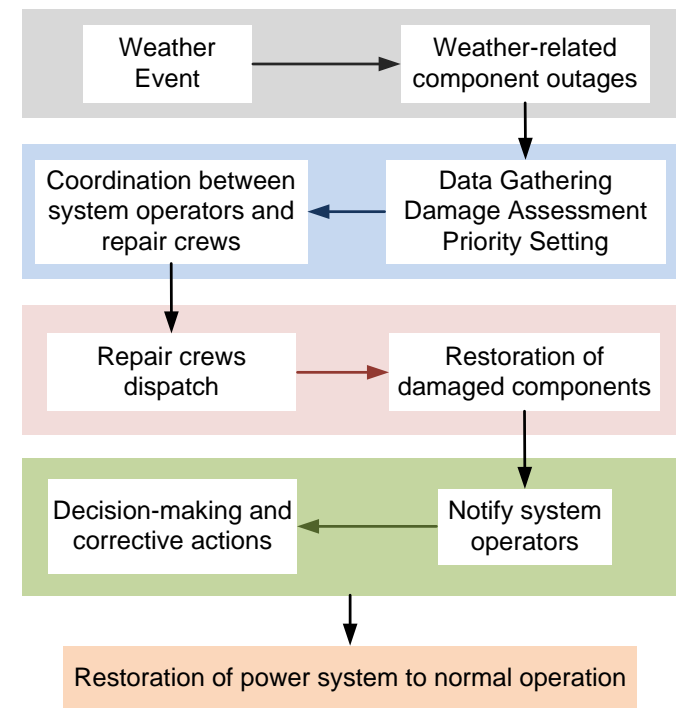

Figure 4: Restoration procedure during weather emergencies

However, this restoration procedure and the recovery time following a weather event depends on several factors, such as the magnitude and location of the event, the availability of spare parts and repair crews and the accessibility to the affected areas. Therefore, similarly to the failure rates, it is critical to 
determine the weather-affected restoration time to increase the accuracy of the modeling output.

There are several approaches to this problem in the literature. In $[39,44,45]$, it is assumed that no repair can take place during the bad weather period because it is argued that it is too dangerous for the repair crews to be dispatched. Considering that additional manpower and resources are employed by the electrical utility during severe weather, a repair rate equal to the one during normal conditions is used in [52]. Billinton and Singh [34] apply three repair scenarios using the two-state and three-state weather models of Figure 1: no repair in adverse weather, repair time equal in normal and adverse weather and repair time 1.5 times the repair time in normal weather. It is concluded, as expected, that assuming no repair in adverse weather results in the highest average outage duration.

In another study by Wang and Billinton [48], weight factors are used to reflect the impact of weather, taking into account the day and time of the restoration. This leads to hourly and weekly time-varying restoration times. It is shown that the time-varying restoration times have a significant impact on the unreliability cost indices. However, the development of these time-varying restoration times requires a vast amount of weather data and system past restoration experience in different weather conditions. Therefore, this results in utility-specific restoration times, and not to a universally applicable restoration model.

A repair rate divider is applied in [33] for which, similarly to the failure rate multiplier discussed earlier, a repair stress in each weather state and the fraction of the line in each weather region are considered. Weather historical data that are associated with each line in the system are, however, required for developing these failure rate multipliers and repair rate dividers.

Ideally, input should be obtained from several electrical utilities on the emergency procedures in place during and after extreme weather events and on the factors governing the restoration times. For example, backup transmission towers may be available which are deployed in case of a weather emergency. This input from the electrical utilities can then serve as a basis for developing a generic restoration model of sufficient accuracy, which could effectively capture the impact of the different weather conditions. 


\subsection{Impact of "human resilience" during weather emergencies}

The critical power infrastructure can be seen as a system-of-systems, for which human response is very important. Dealing with the weather emergency requires the high involvement of system operators in order to monitor and cope with the evolving system conditions. Therefore, system operators' situation awareness, decision-making and response play a key role in preserving system resilience during weather events. In this context, "human resilience" is thus vitally important. In this respect, Mallak [57] defines six factors that drive the level of this human resilience, namely, goal-directed solution seeking, avoidance, critical understanding, role dependence, source reliance and resource access.

It can be appreciated how these high level components defining human resilience can be readily mapped against the requirements of human response (particularly by system operators) under power system stressed conditions. In fact, system operators have to deal with a large amount of data and alarms during electrical and weather emergencies. However, they have certain information processing bottlenecks [5860], which may affect their capability to perceive and interpret the presented data in an effective and timely manner. This influences their reaction to electrical events, which is detrimental to the grid resilience. Panteli et al. [61] show that the insufficient situation awareness and reaction to an electrical event affects significantly the probability of cascading outages and customer disconnection. Nevertheless, the effect of operators' inadequate response during weather emergencies was not evaluated in [61].

Therefore, in addition to the delay in the restoration time due to the weather conditions, the human response can also result in the delayed restoration of the damaged components and of the system to a resilient state. Thus, the main sources of human-related delay during a weather emergency are (Figure 5):

- development of individual situation awareness and decision-making at control centers $(\Delta \mathrm{T}-1)$, and

- coordination and information sharing between the system agents, namely system operators $(\Delta \mathrm{T}-2)$ and repair crew $(\Delta \mathrm{T}-3)$.

Even though the development of smart monitoring and communication technologies has enabled the improvement of situation awareness and the information sharing between the system agents, it is possible 
that due to failures, limitations or design flaws in the information systems or individual operator and repair crew errors, the corrective or preventive actions will not be implemented timely or they will not have the desired effect on the network, compromising the grid resilience. It is therefore critically important to consider these factors in the resilience studies.

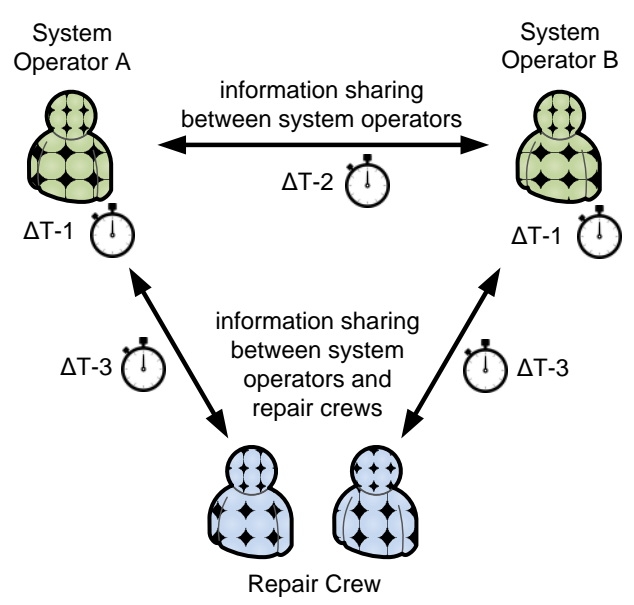

Figure 5: Human-related delay during weather emergencies

\subsection{Independent and common cause failures}

Power systems are traditionally designed to withstand single outages ("N-1" security criterion), but not multiple outages ("N-k" security criterion). However, it is possible that the weather events lead to the simultaneous outage of multiple components if their intensity and/or duration is very high. An example of multiple failures is the outage of a double line circuit due to a collapse of a transmission tower. This is considered a common cause failure, which is evaluated in [44], [52] and [62]. Common cause failures constitute a high threat to the system resilience, as they reduce significantly the system resistance to new outages and also reduce the available assets and resources for coping with the unfolding disturbance.

It is therefore critical to consider both independent and common cause failures in weather-related resilience studies. Resilience enhancement measures can then be applied for mitigating the impact of these events, such as building the transmission towers with more robust materials.

\subsection{Dealing with key modeling uncertainties}

There are several modeling uncertainties when assessing the impact of weather on the resilience of power systems, such as the restoration plans and the potential delay due to the human response, which can 
decrease the accuracy of the modeling output. This is mainly because of the lack of data and of the unpredicted behaviour of the weather. A way of dealing with these modeling uncertainties is performing sensitivity studies to evaluate the impact of the assumptions made in the weather modeling. Different techniques can be used, such as wide range method [63]. For example, in [37] the percentage of failures in adverse weather is varied over a wide range to evaluate its impact on Expected Energy Not Served (EENS). The restoration time is varied in [31] to assess its impact on the system failure rate.

The sensitivity studies would help understand the importance and effect of each modeling aspect and parameter on the modeling output. This will guide the more effective and systematic collection of data that would help increase the accuracy of and the confidence to the simulation output.

\section{Boosting the Resilience of Electrical Networks}

The probability of extreme weather events is currently relatively low, but their impact is so high (and in the future the occurrence frequency might increase due to climate change) that it is vitally important to enhance the resilience of electrical power systems to such events. In this respect, electrical utilities [64, 65] and consulting companies $[66,67]$ worldwide are working towards the development and implementation of grid resilience improvement measures. In fact, hardening to extreme weather has been recognized as one of the key asset management and business goals of several companies, such as [68]. These measures can be divided in short-term, which refer to the preventive and corrective actions before, i.e. days or weeks, during and after the weather event, and long-term, which refer to the long-term planning for providing adaptability and robustness to future weather events and climate change.

\subsection{Short-term resilience measures}

Table 1 shows the short-term resilience measures. These include the traditional preventive and corrective actions for blackout prevention [14], such as reserve planning, generation redispatch and automated protection and control actions, but also additional weather-related measures that can be taken to better prepare for the forthcoming weather event. An accurate forecast of the weather event's location and severity would allow system operators to configure the network in a way to mitigate the impact of the 
severe weather. For example, they can minimize the generation and power flows in the most affected areas to alleviate the post-disturbance line overloadings. Furthermore, the repair and recovery crews would be effectively dispatched and prepositioned to enable the quick restoration of the damaged components [69]. Maintaining backup components and materials also enable the fast replacement of the weather-affected power system components.

During the weather event, it is of key importance to monitor effectively the system state, which enables the development of sufficient situation awareness. This leads to the effective decision-making and reaction by the operators to the electrical events, and also to the efficient coordination with the recovery and repair crews. These crews, weather-permitted, should quickly restore the damaged components to prevent the propagation of the electrical disturbance. The communication infrastructure is critical during the weather event, because it enables the data collection and the communication between the system agents, i.e., system operators and repair crews. It is also critical to monitor the progress of the weather event. If the weather event becomes more intense or follows a different than the predicted route, then the system operators should readjust their emergency plans and possibly apply different corrective and/or preventive actions.

Following the weather event, its impact needs to be assessed and the operators should set priorities for initiating the restoration of the damaged components by the repair crews. The restoration procedures are then applied by the operators for returning the system to a resilient state and reconnecting the customers.

Table 1: Short-term Resilience Measures Before, During and After the Weather Event

Before:

- Accurate estimation of the weather location and severity

- Estimation and prepositioning of the number of repair and recovery crews possibly required following the weather event

- Maintain backup components and materials, such as transmission towers

- Coordination with adjacent networks

- Traditional preventive actions, such as:

- System configuration in a resilient state

- Reserve planning

- Ensure black-start capabilities

- Smart solutions, such as demand-side management

During:

- Monitoring: development of situation awareness 
- Ensure communications functionality

- Coordination with repair and recovery crews

- Traditional corrective actions, such as:

- Generation redispatch

- Substation re-configuration

- Capacitor switching

- Automated protection and control actions: load and generation rejection, system separation, etc.

After:

- Disaster assessment \& priority setting

- Restoration of damaged components, e.g. poles and towers

- Traditional restoration actions, such as:

- Reenergizing transmission lines

- Unit restarting

- Load restoration

- Resynchronization of areas

\subsection{Long-term resilience measures}

Table 2 shows the long-term resilience measures that can be taken for mitigating the influence of weather and providing system adaptability $[1,4,18,19,22,70]$. Following the weather event, the limitations or deficiencies in the operational procedures can be identified. This will enable the improvement of the risk assessment and management, the enhancement of the emergency and preparedness plans and the improvement of vegetation management. In order to be resilient in the face of new emergencies, e.g. unforeseeable weather conditions, an organization should develop robust techniques in problem diagnosis, improvisation, communication and collaborative actions that would enable an effective and fast coordination between the system agents during the weather emergency [71].

Table 2: Long-term Resilience Measures

\begin{tabular}{l}
\hline Operational procedures: \\
- $\quad$ Risk assessment and management \\
- $\quad$ Improve emergency and preparedness plans \\
- $\quad$ Tree trimming/vegetation management for clearing the transmission lines rights-of-way \\
\hline Hardening measures (structural and topology): \\
- $\quad$ Undergrounding distribution and transmission lines \\
- $\quad$ Upgrading poles and structures with stronger, more robust materials \\
- $\quad$ Elevating substations and relocating facilities to areas less prone to flooding \\
- $\quad$ Redundant transmission routes by building additional transmission facilities \\
- $\quad$ Re-routing transmission lines to areas less affected by weather \\
\hline Smart solutions: \\
- $\quad$ Energy storage
\end{tabular}


- Distribution generation

- Demand side management

- Microgrids

- Advanced control and protection schemes, such as System Integrity Protection Schemes (SIPS)

- Advanced visualization and information systems

In addition, structural measures can be taken, such as undergrounding the distribution or transmission lines [4]. This would increase the robustness of the components to severe weather conditions. Furthermore, topology measures, e.g. building additional transmission facilities [4], would improve the assets and resources available to the system operators, which would help manage the disaster as it unfolds.

In the era of smart grids, different smart solutions can be applied for enhancing the grid resilience to extreme weather events. For example, energy storage [72], distributed generation [73] and demand side management [74] can be used for providing flexibility, serving the power consumer without external transmission lines and reducing the power flows in the areas that are highly prone to the severe weather. An additional application of energy storage is the ability to balance microgrids for achieving a balance between generation and demand. Microgrids can separate and isolate themselves from the network during a weather emergency and then automatically resynchronize and reconnect following the weather event.

Advanced protection and control schemes, e.g. System Integrity Protection Schemes (SIPS) [75], can also be used to enhance the grid robustness. These schemes are usually automated and they use fast communications and real-time measurements to adapt their operation on the evolving system conditions, without the need of human intervention. Examples include the load and generation rejection and the system separation. Wide area measurement systems (WAMS) using Phasor Measurement Units (PMUs) can improve the speed and accuracy of these protection and control schemes [75, 76].

The latest technological breakthroughs, including PMUs, can also help improve the visualization and information systems for better supporting the system operators. Available technologies include the intelligent alarm processing for dealing with the vast amount of alarms during emergencies [77], and the advanced visualization techniques such as color contours, animated arrows and dynamically sized pie 
charts, for improving the efficiency of the graphical user interface [78-80].

\subsection{Discussion and Resilience Measures Implications}

In general, there are two critical aspects in the natural disaster emergency and preparedness planning:

what is the system designed to withstand and what can the system owner afford to withstand? The resilience enhancement measures must thus achieve an optimal balance between performance, cost and risk. Therefore, the main drivers of the investments in these measures are:

- improvement of the customer satisfaction,

- enhancement of reliability metrics and safety, and

- reduction or control of the operational and maintenance expenses.

However, some of the measures presented in Tables 1 and 2 are very expensive solutions. In particular, system hardening measures are usually much more expensive than possible operational and "smart grid" solutions. Therefore, the resilience enhancement measures to put in place would ultimately have to be supported by cost benefit analysis that should take account of the possible consequences of the high impact low probability weather events. Before implementing any measures, their cost (including capital, operating and maintenance costs) has to be compared with the reduction in the risk achieved by these measures.

\section{Generic Research Framework For Evaluating the Influence of Weather On Power Systems \\ Resilience}

Based on the discussions of the previous sections, a comprehensive research framework is presented in this section that provides the main modeling aspects and requirements for evaluating the impact of weather on the resilience of power systems. More specifically, the key resilience features of a power system intended as a critical infrastructure, include [9, 10]:

- Robustness/Resistance: This refers to the ability of the system to withstand high-impact low-probability events.

- Resourcefulness: This feature indicates the ability to effectively manage the disaster as it unfolds using the available resources and assets. 
- Response and Recovery: The fast response to and recovery from disruptive events is very important.

- Adaptability: Lessons need to be absorbed from a catastrophe, such as natural hazards, for boosting the key resilience features before the next crisis.

In general, three main models have to be considered, namely, weather, component, and system models (Figure 6). The aim of these models is to provide a suitable quantitative assessment platform to analyze the impact of weather on the key resilience features outlined above. In this light, these models interact with each other for obtaining the weather-affected system resilience indices. As can be seen in Figure 6, the weather model provides input to both the component and system models. The component model uses the input of the weather model to estimate the weather-adjusted component resilience and operation, which is used as input to the system model. Then, in the system analysis, the inputs from the weather and component models are used for carrying out the performance assessment of the system in order to estimate the effect of weather on the system resilience indices.

\subsection{Weather model}

Developing a highly accurate weather profile is important in such studies. Historical weather observations or recorded data can be used for creating the regional weather profiles. These can be obtained from measurements at the weather stations of each country or from site measurements at different locations in the system, such as wind parks or transmission lines and towers. Alternatively, weather generators can be used for generating the weather data required. For example, the UKCP09 weather generator [81] developed by the Met Office, UK [82] is a free software that uses historical weather data for training the weather generator, which then provides long time series of weather data using random number sampling. An additional example includes the Hazard U.S. Multi-Hazard (Hazus-MH) tool [83] provided by the Federal Emergency Management Agency (FEMA) [84], which generates natural hazard scenarios, and in particular earthquakes, hurricanes and floods. If sufficient real observation data or such weather generators are not available, simulation techniques can support the generation of the weather profile, as in [49], [50] and [85], which should be capable to capture the stochastic and continuously varying behaviour of the weather. 
However, these have to be accompanied by methods for reducing or evaluating the influence of the weather forecast errors on the accuracy of the simulation output, such as [86]. Finally, the weather profiles should be time- and space-domain, with the time resolution ideally being half hourly or hourly.

As aforementioned, the climate change is expected to increase the frequency, severity and duration of extreme weather events. Future weather scenarios are thus needed for capturing this impact of climate change on the resilience of future power systems. These scenarios can be obtained from the weather generators discussed above. The resilience assessment using future weather scenarios would provide an indication of the measures to be applied for boosting the future grid resilience.

In addition to the weather profiles, the weather model should be able to provide the relation between the weather and the components' resilience and operation. An example includes the fragility curves discussed in Section 3.3. This can be achieved by using historical weather data and associating the behaviour of the components, e.g. outage, derated operation, etc., with these weather events, as in [87]. This will provide a statistical impact of weather on the failure frequency and operation of power system components, which can be used to model the behaviour of the components during extreme weather events.

\subsection{Component model}

The weather conditions that power system components experience affect significantly their reliability, i.e. failure and restoration rates, and operation. Using the input from the weather model on the relation between weather and components' resilience and operation, the component model should be capable to model this impact of weather while taking into account the structural, e.g. material, and operational, e.g. operating temperature, characteristics of the components.

Additionally, the component model should be flexible and easily modified, in order to incorporate any future changes in the component characteristics. It can be a function of the parameters affecting the resilience and operation of the component, such as material, geographical location, height, temperature and wind speed and direction. A generic model for each type of components can be developed, e.g. transmission lines or transmission towers, which should be easily adjusted to specific components 
depending on the components' characteristics and on the impact of weather on each component.

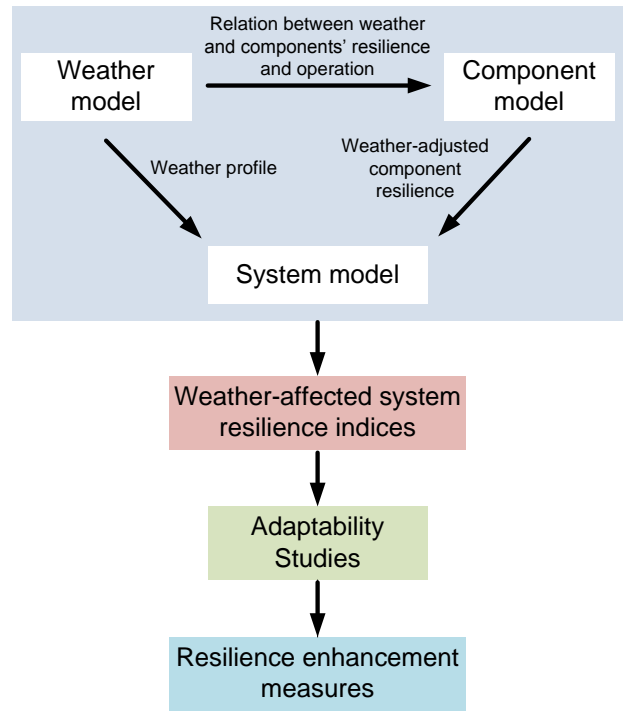

Figure 6: Generic research framework for assessing the impact of weather on power systems resilience

In addition to the impact of weather on components' resilience, the effect on components' operation should be included in the component model as it affects the operation of the entire power infrastructure. For example, when modeling the wind turbines, the cut-in and cut-off wind speeds should be considered. This is particularly critical when modeling extreme wind speed events, typically higher than $25 \mathrm{~m} / \mathrm{s}$.

Furthermore, the component model should be capable to capture the continuous varying weather conditions, without the need of distinguishing normal and bad weather. In this way, there will not be a different model for each weather state, but only one universal model for any weather conditions which reduces the complexity of the simulation procedure.

In general, the operational reliability of a component is affected by the weather conditions and the loading conditions [88, 89]. The effect of weather has been extensively discussed in this paper. In conventional reliability evaluation of power systems, it is assumed that the failure rates of the components are constant and loading-independent. However, in real-time operation, the components experience higher stress during heavily loading conditions, which affects their failure probability. Therefore, the dependency between components' reliability and loading needs to be considered. Developing a comprehensive model using weather- and loading-dependent reliability data would provide a clearer indication of the operational 
reliability of power systems.

\subsection{System model}

The weather profile and the weather-adjusted component resilience and operation are then used by the system model for assessing the performance of the system under different weather conditions. For this purpose, as discussed in Section 3.1., it is preferable to use time series simulation techniques. This enables the modeling of a weather event in both time and space domain, which helps capture the stochastic nature and impact of weather while moving across the transmission network. The time series weather profile can then be mapped to the component model for obtaining the operational state of the components at every simulation step. It is also vitally important to take into account the spatial characteristics of the weather event, because the transmission network covers a large geographic area, so the effect of weather would vary from region to region.

Power systems are by definition system-of-systems, so by affecting the robustness of the individual components, the weather events can initiate cascading impacts on the entire infrastructure. Therefore, the output of the system model should provide a clear indication of the impact of weather on the resilience features of the entire power system. In addition, using the weather regions discussed in Section 3.2., a regional indication of the risk introduced by severe weather events can be estimated, which will provide a regional vulnerability index that will help target the resilience enhancement measures to the most vulnerable regions of the system.

Even though assessing the impact of future climate change on the reliability of power systems using the current system topologies, operational procedures and protection and control methods provides an indication of the problem, it does not accurately reflect either the real behaviour of future networks or the extend of the problem in the future. When modeling the influence of future weather scenarios, it is more realistic to take into account the practices of future networks and future demand scenarios. The latest trends within the concept of smart grids show that power systems are experiencing a transition from the conventional way of operating and protecting the grid to a smarter and more adaptive way. Technologies 
and practices, such as energy storage, demand side management, microgrids, adaptive wide area protection schemes and decentralized protection and control, are going to affect the operation and reliability of future networks. In addition, several technological developments, such as electrical vehicles and increased appliances efficiency, would result in significant changes in the typical existing demand patterns. Also, the progress in the monitoring and visualization tools would enhance the observability of the system, resulting in a higher operators' situation awareness and response to electrical events. Therefore, these aspects have to be included in the resilience studies in order to obtain a complete and more accurate indication of the problem in the future, which will drive the decision on the most suitable resilience enhancement measures.

Finally, several indices can be used, such as Expected Energy Not Served (EENS) and Loss of Load Probability (LOLP) [90]. For evaluating the effect of the modeling parameters on these reliability indices, sensitivities studies can be performed as discussed in Section 3.7.

\subsection{Adaptability studies and resilience enhancement measures}

Following the estimation of the weather effects on the resilience of power systems, adaptability studies should be conducted, whose aim should be the enhancement of the key resilience features. This will help prevent or mitigate the impact of future weather events. In fact, it is critically important for a power system to adapt to the future weather conditions in order to cope with the possible increase in the frequency, duration and/or intensity of extreme weather phenomena, possibly engendered by climate change.

Based on the outcome of the adaptability studies, resilience enhancement measures can be applied, as discussed in Section 4. The effect of these measures on the key resilience features varies. For example, upgrading the components with stronger materials makes them more robust to the weather conditions, which reduces their probability of failure. Effective operational procedures and enhanced situation awareness help reduce the restoration time, which improves the recovery feature of resilience. Redundant transmission facilities and smart grid solutions provide additional resources and control assets to the system operators, which help them cope effectively with the unfolding disaster. This altogether improves the resourcefulness feature of resilience. 


\section{Conclusions}

Severe weather has a significant impact on the critical power infrastructure. In combination with the potential increase in the frequency, severity and duration of such events due to the climate change, boosting the grid resilience to extreme weather phenomena becomes increasingly important.

Quantifying the risk introduced by weather is a difficult task due its high stochasticity and multidimensional impact. A review of existing methodologies for assessing the impact of weather on the resilience of power systems is presented in this paper. This helps identify the key modeling approaches, requirements and challenges in such studies, e.g., modeling the impact of weather on the failure and restoration rates of power system components, which is directly relevant to the concept of resilience.

Several resilience enhancement measures are then discussed in the paper. These can be divided in short-term, i.e., before, during and after the weather emergency, and long-term for providing robustness and adaptability to future weather conditions. Preventive control actions and proper operational procedures help prepare for the forthcoming weather event, while corrective actions help manage effectively the disaster as it unfolds. In addition, structural and topology measures increase the resistance and resourcefulness of the system to extreme weather events. Smart grid solutions can also be applied for providing flexibility and advanced monitoring, protection and control strategies to mitigate the effect of severe weather. In addition to what is discussed in the existing body of literature, it has been argued in this paper that the resilience of the critical power infrastructure should be modeled and assessed within the context of system-of-systems that also include human response as a key dimension.

Based on this review and the relevant emerging considerations, a generic research framework is proposed which helps model the impact of any weather conditions on any power system. It is mainly composed of three models, namely, the weather, component, and system models, which interact with each other for obtaining the weather-affected system resilience indices. This framework can be used as the basis for developing weather-related resilience studies, as it discusses and incorporates the key modeling aspects when evaluating the effect of weather on power systems resilience. 


\section{Acknowledgments}

This work was supported by the Resilient Electricity Networks for Great Britain (RESNET) project, which

is funded by the Engineering and Physical Sciences Research Council (EPSRC), UK.

\section{References}

[1] R.J. Campbell, Weather-Related Power Outages and Electric System Resiliency, 2012.

[2] A. Kenward, U. Raja, Blackout: Extreme Weather, Climate Change and Power Outages, 2014.

[3] Witt Associates, Connecticut October 2011 Snowstorm Power Restoration Report, 2011.

[4] Executive Office of the President, Economic Benefits of Increasing Electric Grid Resilience to Weather Outages, 2013.

[5] Intergovernmental Panel on Climate Change (IPCC), Managing the Risks of Extreme Events and Disasters to Advance Climate Change Adaptation, 2012.

[6] UK Energy Research Center (UKERC), Building a Resilient UK Energy System, 2009.

[7] UK Energy Research Center (UKERC), Energy 2050 project, 2009.

[8] Power Systems Engineering Center (PSERC), Engineering Resilient Cyber-Physical Systems, 2012.

[9] National Infrastructure Advisory Council (NIAC), A Framework for Establishing Critical Infrastructure Resilience Goals, 2010.

[10] Cabinet Office, Keeping the Country Running: Natural Hazards and Infrastructure, 2011.

[11] The National Association of Regulatory Utility Commissioners (NARUC), Resilience in Regulated Utilities, 2013.

[12] DNV GL, Adaptation to a changing climate, 2014.

[13] D. Ward, The effect of weather on grid systems and the reliability of electricity supply, Climatic Change 121 (2013) 103-113.

[14] U.G. Knight, Power Systems in Emergencies: From Contingency Planning to Crisis Management, John Wiley and Sons Ltd, Chichester, 2001.

[15] M. Bruch, V. Munch, M. Aichinger, M. Kuhn, M. Weymann, G. Schmid, Power Blackout Risks, 2011.

[16] A. Shafieezadeh, U.P. Onyewuchi, M.M. Begovic, R. DesRoches, Age-Dependent Fragility Models of Utility Wood Poles in Power Distribution Networks Against Extreme Wind Hazards, IEEE Trans. Power Deliv. 29 (2014) 131-139.

[17] Asian Development Bank, Climate Risk and Adaptation in the Electric Power Sector, 2012.

[18] National Grid Electricity Transmission PLC, Climate Change Adaptation Report, 2010.

[19] Energy Networks Association, Electricity Networks Climate Change Adaptation Report, 2011.

[20] VTT, Recognizing climate change in electricity network design and construction, 2007.

[21] L.M. Beard, J.B. Cardell, I. Dobson, F. Galvan, D. Hawkins, W. Jewell, et al., Key Technical Challenges for the Electric Power Industry and Climate Change, IEEE Trans. Energy Convers. 25 (2010) 465-473.

[22] Northern PowerGrid, Climate Change Adaptation Report.

[23] Univ. of Cambridge and World Energy Council, Climate Change: Implications for the Energy Sector, 2013.

[24] R. Schaeffer, A.S. Szklo, A.F. Pereira de Lucena, B.S. Moreira Cesar Borba, L.P. Pupo Nogueira, F.P. Fleming, et al., Energy sector vulnerability to climate change: A review, Energy 38 (2012) 112.

[25] S. Mirasgedis, Y. Sarafidis, E. Georgopoulou, V. Kotroni, K. Lagouvardos, D.P. Lalas, Modeling framework for estimating impacts of climate change on electricity demand at regional level: Case of Greece, Energy Convers. Manag. 48 (2007) 1737-1750. 
[26] M. Ruth, A.C. Lin, Regional energy demand and adaptations to climate change: Methodology and application to the state of Maryland, USA, Energy Policy 34 (2006) 2820-2833.

[27] D.J. Sailor, J.R. Muñoz, Sensitivity of electricity and natural gas consumption to climate in the U.S.A. - Methodology and results for eight states, Energy 22 (1997) 987-998.

[28] S.J. Parkpoom, G.P. Harrison, Analyzing the Impact of Climate Change on Future Electricity Demand in Thailand, IEEE Trans. Power Syst. 23 (2008) 1441-1448.

[29] S. Parkpoom, G.P. Harrison, J.W. Bialek, Climate change impacts on electricity demand, in: Proc. of the Universities Power Engineering Conference (UPEC), 2004.

[30] D.P. Gaver, F.E. Montmeat, A.D. Patton, Power System Reliability I-Measures of Reliability and Methods of Calculation, IEEE Trans. Power App. Syst. 83 (1964) 727-737.

[31] R. Billinton, K.E. Bollinger, Transmission System Reliability Evaluation Using Markov Processes, IEEE Trans. Power App. Syst. PAS-87 (1968) 538-547.

[32] R. Billinton, C. Wu, G. Singh, Extreme adverse weather modeling in transmission and distribution system reliability evaluation, in: Proc. of the Power Systems Computational Conference (PSCC), 2002.

[33] M.R. Bhuiyan, R.N. Allan, Inclusion of weather effects in composite system reliability evaluation using sequential simulation, IEE Proc. of Gener., Trans. and Distr. 141 (1994) 575-584.

[34] R. Billinton, G.D. Singh, Reliability assessment of transmission and distribution systems considering repair in adverse weather conditions, in: Proc. of the IEEE Canadian Conference on Electrical and Computer Engineering (CCECE), 2002

[35] Y. Liu, C. Singh, Reliability Evaluation of Composite Power Systems Using Markov Cut-Set Method, IEEE Trans. Power Syst. 25 (2010) 777-785.

[36] R. Billinton, R.N. Allan, Reliability Evaluation of Power Systems, Plenum Press, New York, 1996.

[37] R. Billinton, L. Wenyuan, A novel method for incorporating weather effects in composite system adequacy evaluation, IEEE Trans. Power Syst. 6 (1991) 1154-1160.

[38] R. Billinton, L. Cheng, Incorporation of weather effects in transmission system models for composite system adequacy evaluation, IEE Proc. of Gener., Trans. and Distr. 133 (1986) 319-327.

[39] R. Billinton, G. Singh, Application of adverse and extreme adverse weather: modelling in transmission and distribution system reliability evaluation, IEE Proc. of Gener., Trans. and Distr. 153 (2006) 115-120.

[40] IEEE, Terms for reporting and analyzing outages of electrical transmission and distribution facilities and interruptions to customer service, 1973.

[41] R. Billinton, J. Acharya, Consideration of multi-state weather models in reliability evaluation of transmission and distribution systems, in: Proc. of the IEEE Canadian Conference on Electrical and Computer Engineering (CCECE), 2005.

[42] A.F. Mensah, L. Dueñas-Osorio, Outage Predictions of Electric Power Systems under Hurricane Winds by Bayesian Networks, in: Proc. of the $13^{\text {th }}$ Intern. Conf. on Probabilistic Methods Applied to Power Systems (PMAPS), in press, 2014.

[43] Y. Zhou, A. Pahwa, S. Yang, Modeling Weather-Related Failures of Overhead Distribution Lines, IEEE Trans. Power Syst. 21 (2006) 1683-1690.

[44] Y. Liu, C. Singh, Evaluation of hurricane impact on composite power system reliability considering common-cause failures, Int. J. Syst. Assur. Eng. Manag. 1 (2010) 135-145.

[45] Y. Liu, C. Singh, A Methodology for Evaluation of Hurricane Impact on Composite Power System Reliability, IEEE Trans. Power Syst. 26 (2011) 145-152.

[46] A. Islam, Smart Grid Reliability Assessment Under Variable Weather Conditions, Doctor of Philosophy, Department of Electrical Engineering, University of South Florida, 2009.

[47] M.A. Rios, D.S. Kirschen, D. Jayaweera, D.P. Nedic, R.N. Allan, Value of security: modeling timedependent phenomena and weather conditions, IEEE Trans. Power Syst. 17 (2002) 543-548.

[48] P. Wang, R. Billinton, Reliability cost/worth assessment of distribution systems incorporating timevarying weather conditions and restoration resources, IEEE Trans. Power Deliv. 17 (2002) 260-265. 
[49] K. Alvehag, L. Soder, A Stochastic Weather Dependent Reliability Model for Distribution Systems, in: Proc. of the $10^{\text {th }}$ Intern. Conf. on Probabilistic Methods Applied to Power Systems (PMAPS), 2008.

[50] K. Alvehag, L. Soder, A Reliability Model for Distribution Systems Incorporating Seasonal Variations in Severe Weather, IEEE Trans. Power Deliv. 26 (2011) 910-919.

[51] G. Li, P. Zhang, P.B. Luh, W. Li, Z. Bie, C. Serna, et al., Risk Analysis for Distribution Systems in the Northeast U.S. Under Wind Storms, IEEE Trans. Power Syst. 29 (2014) 889-898.

[52] R. Barben, Vulnerability Assessment of Electric Power Supply under Extreme Weather Conditions, Doctor of Philosophy, Doctor of Philosophy, Laboratoire Des Systemes Energetiques, Ecole Pytechnique Federale De Laussane, Laussane, 2010.

[53] G.C. Marano, R. Greco, M. Mezzina, Stochastic Approach for Analytical Fragility Curves, KSCE J. Civil Eng. 12 (2008) 305-312.

[54] Resilient Electricity Networks for Great Britain (RESNET). Available from: http://www.ncl.ac.uk/ceser/researchprogramme/systemsmodelling/resnetresilientelectricitynetworks Last Accessed: October 2013.

[55] M. Sbinozuka, M. Feng, X. Dong, S.E. Chang, T.C. Cheng, X. Jin, et al., Advances in Seismic Performance Evaluation of Power Systems, 2003.

[56] J. Winkler, L. Dueñas-Osorio, R. Stein, D. Subramanian, Performance assessment of topologically diverse power systems subjected to hurricane events, Reliab. Eng. Syst. Saf. 95 (2010) 323-336.

[57] L.A. Mallak, Measuring resilience in health care provider organizations, Health Manpower Manag. 24 (1998) 148-152.

[58] M.R. Endsley, B. Bolte, D.G. Jones, Designing for situation awareness: An approach to usercentered design, Taylor and Francis Group, Boca Ranton, 2003.

[59] M.R. Endsley, E.S. Connors, Situation awareness: State of the art, in: Proc. of the IEEE Power and Energy Society General Meeting - Conversion and Delivery of Electrical Energy in the $21^{\text {st }}$ Century, 2008.

[60] E.S. Connors, M.R. Endsley, L. Jones, Situation Awareness in the Power Transmission and Distribution Industry, in: Proc. of the Human Factors and Ergonomics Society 31st Annual Meeting, 2007.

[61] M. Panteli, P.A. Crossley, D.S. Kirschen, D.J. Sobajic, Assessing the Impact of Insufficient Situation Awareness on Power System Operation, IEEE Trans. Power Syst. 28 (2013) 2967-2977.

[62] R. Billinton, Y. Kumar, Transmission Line Reliability Models Including Common mode and Adverse Weather Effects, IEEE Trans. Power App. Syst. PAS-100 (1981) 3899-3910.

[63] IEEE PES Nuclear Power Engineering Committee, IEEE Guide for General Principles of Reliability Analysis of Nuclear Power Generating Station Safety Systems, 1985.

[64] NERC, Severe Impact Resilience: Considerations and Recommendations, 2012.

[65] NERC, High-Impact Low-Frequency Event Risk to the North American Bulk Power System, 2010.

[66] Quanta Technology, Extreme Weather Event Analyses \& System Hardening, 2013.

[67] Quanta Technology, Hardening Transmission and Distribution Systems Against Extreme Weather Events, 2013.

[68] Quanta Technology, Available from: http://www.quanta-technology.com/expertise-areas/assetmanagement, Last Accessed: June 2014.

[69] E.V. Badolato, J. Bleiweis, J.D. Craig, J. Horace W. Fleming, Hurricane Hugo: Lessons Learned in Energy Emergency Preparedness, 1990.

[70] B. Fleming, Climate Change Adaptation Report, 2011.

[71] H.B. Leonard, A.M. Howitt, Routine or Crisis: The Search for Excellence, Crisis Response 4 (2008) 32-35.

[72] P.F. Ribeiro, B.K. Johnson, M.L. Crow, A. Arsoy, Y. Liu, Energy storage systems for advanced power applications, IEEE Proceedings 89 (2001) 1744-1756. 
[73] J.A.P. Lopes, N. Hatziargyriou, J. Mutale, P. Djapic, N. Jenkins, Integrating distributed generation into electric power systems: A review of drivers, challenges and opportunities, Electr. Power Syst. Res. 77 (2007) 1189-1203.

[74] G. Strbac, Demand side management: Benefits and challenges, Energy Policy 36 (2008) 4419-4426.

[75] V. Madani, D. Novosel, S. Horowitz, M. Adamiak, J. Amantegui, D. Karlsson, et al., IEEE PSRC Report on Global Industry Experiences With System Integrity Protection Schemes (SIPS), IEEE Trans. Power Deliv. 25 (2010) 2143-2155.

[76] A.G. Phadke, J.S. Thorp, Synchronized Phasor Measurements and Their Applications, Springer, New York, 2008.

[77] D.S. Kirschen, B.F. Wollenberg, Intelligent alarm processing in power systems, IEEE Proceedings 80 (1992) 663-672.

[78] J.D. Weber, T.J. Overbye, Voltage contours for power system visualization, IEEE Trans. Power Syst. 15 (2000) 404-409.

[79] T.J. Overbye, J.D. Weber, Visualizing the electric grid, IEEE Spectrum 38 (2001) 52-58.

[80] R.P. Klump, J.D. Weber, Real-time data retrieval and new visualization techniques for the energy industry, in: Proc. of the $35^{\text {th }}$ Annual Hawaii International Conference on System Sciences (HICSS), 2002.

[81] Met Office UK, What is UKCP09?, Available from: http://ukclimateprojections.metoffice.gov.uk/, Last Accessed: November 2013.

[82] Met Office UK, Available from: http://www.metoffice.gov.uk/, Last Accessed: November 2013.

[83] Federal Emergency Management Agency (FEMA), Hazards U.S. Multi-Hazard (HAZUS-MH), Available from: http://www.csc.noaa.gov/digitalcoast/tools/hazus-mh, Last Accessed: April 2014.

[84] Federal Emergency Management Agency (FEMA), Available from: http://www.fema.gov/hazus, Last Accessed: April 2014.

[85] P.J. Vickery, F.J. Masters, M.D. Powell, D. Wadhera, Hurricane hazard modeling: The past, present, and future, J. Wind Eng. Ind. Aerodyn. 97 (2009) 392-405.

[86] D. Fay, J.V. Ringwood, On the Influence of Weather Forecast Errors in Short-Term Load Forecasting Models, IEEE Trans. Power Syst. 25 (2010) 1751-1758.

[87] K. Murray, K.R.W. Bell, Wind Related Faults on the GB Transmission Network, in: Proc. of the $13^{\text {th }}$ Intern. Conf. on Probabilistic Methods Applied to Power Systems (PMAPS), in press, 2014.

[88] Y. Sun, P. Wang, L. Cheng, H. Liu, Operational reliability assessment of power systems considering condition-dependent failure rate, IET Gener., Trans. and Distr. 4 (2010) 60-72.

[89] F. Weihui, J.D. McCalley, V. Vittal, Risk assessment for transformer loading, IEEE Trans. Power Syst. 16 (2001) 346-353.

[90] R. Billinton and W. Li, Reliability Assessment of Power Systems Using Monte Carlo Methods, Plenum Press, New York, 1994. 